\title{
A new algorithm for color image comparison based on similarity measures
}

\author{
Daniel Paternain, Mikel Galar, Aranzazu Jurio, Edurne Barrenechea \\ Departamento de Automatica y computacion \\ Universidad Publica de Navarra \\ 31006 Pamplona, Spain
}

\begin{abstract}
In this work we address the problem of the quality assessments in the process of color images segmentation. We consider each component of a color image as a fuzzy set and therefore, we propose to use similarity measures (between fuzzy sets) to compare image segmentations. We test three segmentation algorithms, FCM [2], MAP-ML [11] and 2-TUP [21] on Berkeley segmentation database [17] and we evaluate the obtained results using our proposal.
\end{abstract}

Keywords: Similarity measure, Image segmentation, Image comparison

\section{Introduction}

A key problem for vision systems is the identification of sub-images (which represent objects) on an image. For human observers this operation could be very simple, but very difficult for machines (see [22]). The division of an image into regions is called segmentation. Actually, the segmentation of digital images is the process of dividing an image into disjoint parts, regions or classes so that each one of them has very concrete attributes or properties. Each of these classes represents an object of the image.

There exist different measures to compare the goodness of a segmentation algorithm $[1,17,18$, 25]. A very useful mechanic is the possibility of comparing two segmented images, one obtained by an approach and the other one hand-made (denoted as ideal image).

In [17] different ideal images are provided by experts. One factor that crucially affects to image segmentation is the number of classes given by the experts since it depends on the evaluation criteria (color, shape, texture, etc.). In addition, depending on the context or application, experts can perform different segmentations. Taking into account the hypothesis that the pixels belonging to an object have a similar color, we consider necessary to propose a comparison algorithm that allows us to calculate how similar two segmentations of a given color image are. Obviously, if one of these segmentations is the ideal image, then our comparison algorithm allows us to objectively measure the goodness of the segmentation obtained by any segmentation method. The main advantage of our proposal is that it does not penalize if one pixel is classified in different objects in the two segmentations, as long as the mean (average) color of those object are similar.

It is well known that digital images themselves include an important degree of uncertainty. For this reason, Fuzzy Set Theory [28] has been widely used as a tool to deal with problems in image processing field $[3,4,7,10,15]$. This is due to limitations of the discrete grayscale/color used in the codification or the sampling process done to fit the analogue image into a pixel matrix. Consequently, some information about the real-world image is always lost in the digitalization process. For these reasons, among others, our proposal uses Fuzzy Set Theory.

The algorithm we provide in this work is based on: (a) the representation of a color image RBG by means of fuzzy sets, one fuzzy set for each color component, and (b) fuzzy similarity measures.

The work is organized as follows: Section 2 recalls some basic concepts. In Section 3 we present the algorithm for image color comparison. Next, in Section 4 we show the results obtained with our proposal and we compare them with other approaches. Some conclusions are raised in Section 5.

\section{Preliminaries}

In this section we recall some results that are the basis of our proposal to compare segmented color images.

Definition 1 [28] A fuzzy set $A$ on a finite universe $U$ is a mapping $U \rightarrow[0,1]$.

We will denote by $\mathcal{F} S(U)$ the set of all the fuzzy sets on $U$ and by $\operatorname{Card}(A)$ the cardinal of the fuzzy set $A \in \mathcal{F} S(U)$.

We know that in fuzzy set theory a function $c$ : $[0,1] \rightarrow[0,1]$ such that $c(0)=1, c(1)=0$ that is strictly decreasing and continuous is called strict negation. If, in addition, $\mathrm{c}$ is involutive, then it is said that it is a strong negation.

Bustince et al. [5] define the concept of Restricted Equivalence Function ( $R E F)$. This concept arises on the one hand, from the definition of equivalence 
given by Fodor and Roubens [13], and on the other, from the properties usually demanded from the measures used for comparing images (see $[9$, $10,26,27])$. The authors also present different construction methods of REFs from automorphisms and implication operators. They apply REFs to the computation of the threshold of a gray scale image [6].

Orduna et al. [21] address the problem of color image segmentation transforming it into a decision making paradigm. They consider a set of experts, so that each expert assigns a preference degree of each pixel to every object of the image (using REFs) and taking into account also the ignorance associated to such assignation [8, 24]. Finally, the authors represent the objects by means of fuzzy linguistic labels and using the decision-making model based on 2-tuples [14] each pixel is classified.

Definition 2 [5] A function REF : [0, 1] $]^{2} \rightarrow[0,1]$ is called restricted equivalence function, associated to a strong negation $c$, if it satisfies the following conditions:

1) $R E F(x, y)=R E F(y, x)$ for all $x, y \in[0,1]$;

2) $R E F(x, y)=1$ if and only if $x=y$;

3) $\operatorname{REF}(x, y)=0$ if and only if $x=1$ and $y=0$ or $x=0$ and $y=1$;

4) $R E F(x, y)=R E F(c(x), c(y))$ for all $x, y \in$ $[0,1]$;

5) For all $x, y, z \in[0,1]$, if $x \leq y \leq z$, then $\operatorname{REF}(x, y) \geq \operatorname{REF}(x, z)$ and $\operatorname{REF}(y, z) \geq$ $R E F(x, z)$.

Next, we recall a construction method of REFs from automorphisms.

Definition 3 We will call automorphism of the unit interval every function $\varphi:[0,1] \rightarrow[0,1]$ that is continuous and strictly increasing such that $\varphi(0)=$ 0 and $\varphi(1)=1$.

Proposition 1 [5] If $\varphi_{1}, \varphi_{2}$ are two automorphisms of the unit interval, then,

$$
R E F(x, y)=\varphi_{1}^{-1}\left(1-\left|\varphi_{2}(x)-\varphi_{2}(y)\right|\right)
$$

with $c(x)=\varphi_{2}^{-1}\left(1-\varphi_{2}(x)\right)$ is a restricted equivalence function.

Example 1 Let $\varphi_{1}(x)=\varphi_{2}(x)=x$, then

$$
\operatorname{REF}(x, y)=1-|x-y|
$$

is a restricted equivalence function.

Proposition 2 [5] In the conditions of Proposition 1

$$
\begin{gathered}
\operatorname{REF}(1, x)=x \text { for all } x \in[0,1] \\
\text { if and only if } \\
\varphi_{1}(x)=\varphi_{2}(x) \text { for all } x \in[0,1]
\end{gathered}
$$

Besides, the authors propose a method for constructing similarity measures in the sense of Liu ([16]) and proximity measures in the sense of Fan and Xie ([12]). We recall the construction method of these measures described in [5].

Proposition 3 [5] Let $\mathcal{M}:[0,1]^{n} \rightarrow[0,1]$ be such that it fulfills:

(A1) $\mathcal{M}\left(x_{1}, \ldots, x_{n}\right)=0$ if and only if $x_{1}=\cdots=$ $x_{n}=0$,

(A2) $\mathcal{M}\left(x_{1}, \ldots, x_{n}\right)=1$ if and only if $x_{1}=\cdots=$ $x_{n}=1$,

(A3) $\mathcal{M}$ is nondecreasing;

Let $R E F:[0,1]^{2} \rightarrow[0,1]$ be a restricted equivalence function. Under these conditions

$$
\begin{gathered}
S M: F S(U) \times F S(U) \rightarrow[0,1], \text { given by } \\
S M(A, B)=\mathcal{M}_{i=1}^{n} R E F\left(A\left(u_{i}\right), B\left(u_{i}\right)\right),
\end{gathered}
$$

it satisfies the following items:

(i) $S M(A, B)=S M(B, A)$, for all $A, B \in \mathcal{F}(U)$;

(ii) $S M\left(A, A_{c}\right)=0$, for all non fuzzy set $A$;

(iii) $S M(A, B)=1$, if and only if $A=B$;

(iv) If $A \leq B \leq C$, then $\operatorname{SM}(A, B) \geq S M(A, C)$ and $S M(C, B) \geq S M(C, A)$.

(iv) $S M\left(A_{c}, B_{c}\right)=S M(A, B)$

Example 2 If we take $\mathcal{M}$ as the arithmetic mean aggregation and $\operatorname{REF}(x, y)=1-|x-y|$ then by Proposition 3 we have that for all $A, B \in F S(U)$

$$
S M(A, B)=\frac{1}{n} \sum_{i=1}^{n} 1-\left|A\left(u_{i}\right)-B\left(u_{i}\right)\right| .
$$

When Eq. (3) is used for global comparison of two images it is called similarity measure based on contrast de-enhancement [10].

\section{A new approach to compare segmented color images}

In the literature, there exist different methods to segment color images $([2,11,19,20])$. Evidently, a very important key point is to establish a measure for evaluating the quality of the results obtained with these different approaches. Apart from a visual comparison, it is necessary to carry out a quantitative comparison. The most intuitive methods for the evaluation of segmented images, and a priori the most objective ones, are those that compare the obtained solution with the ideal segmentation for that image.

This ideal segmentation is usually hand-made, but depending on the context of application, experts can perform different segmentations. One of the criteria that is affected by the purpose of the segmentation is the number of classes, but the results also vary strongly depending on whether 
other characteristics like color, shape or texture are considered or not.

In this work we present a new algorithm for comparing color image segmentations. It is only based on the color of the segmented areas. In this sense, if in an image there are two objects with a similar color that in the ideal segmentation are labeled as different objects, the error classifying both as the same object is very low. For example, in Figure 3 we show two horses. The ideal segmentation made by an expert can separate them as two different objects. If we evaluate a segmentation where they are labeled as the same object, our proposal will get low error, because their color is quite similar.

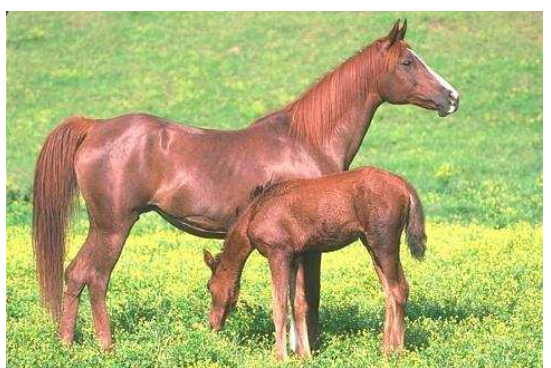

Our proposal is based on the Representative Color of each object of the segmented images to be compared. We define the Representative Color as the mean color of all the pixels labeled as the same object. Each color component $(R, G, B)$ is calculated as the average of the intensities of the considered pixels in that component. We propose a new index called Total Comparison Index , $C I_{\text {Total }}$, that measure how similar two segmented images are. It is calculated as the difference between the Representative Color of every pixel in both images.

\subsection{Comparison algorithm}

Let $Q$ be an image in the RGB color space; that is, $Q=\left(Q_{R}, Q_{G}, Q_{B}\right)$ where $Q_{R}, Q_{G}, Q_{B}$ are red, green and blue components, respectively. In this way, a color image of $N \times M$ pixels is a collection of $N \times M \times 3$ elements arranged in rows and columns. For each component, each pixel is assigned with a numerical value in $\{0,1, \cdots, L-1\}$, representing its intensity. For us, each component $Q_{i}$, being $i \in\{R, G, B\}$, is represented by a fuzzy set, where the membership degree is the normalized intensity. Therefore $Q \in \mathcal{F} S(U)^{3}$.

The scheme of our proposed comparison algorithm is given in Algorithm 1. It is divided in three main steps. The first one consists in creating two new images associated with the two segmented ones. In both of them, each area is labeled with its Representative Color. Based on these new images, we calculate, for each color component, the Comparison Index. This is a similarity measure between both images. In this sense, if both segmentations are equal, the similarity takes 1 , the maximum value. Finally, we aggregate by the arithmetic mean all the comparison indexes, obtaining the Total Comparison Index, that is the final value returned by the algorithm.

It is necessary to remark that the number of classes of both segmented images to compare may be different. Even the original image can be interpreted as a special case of segmented image.

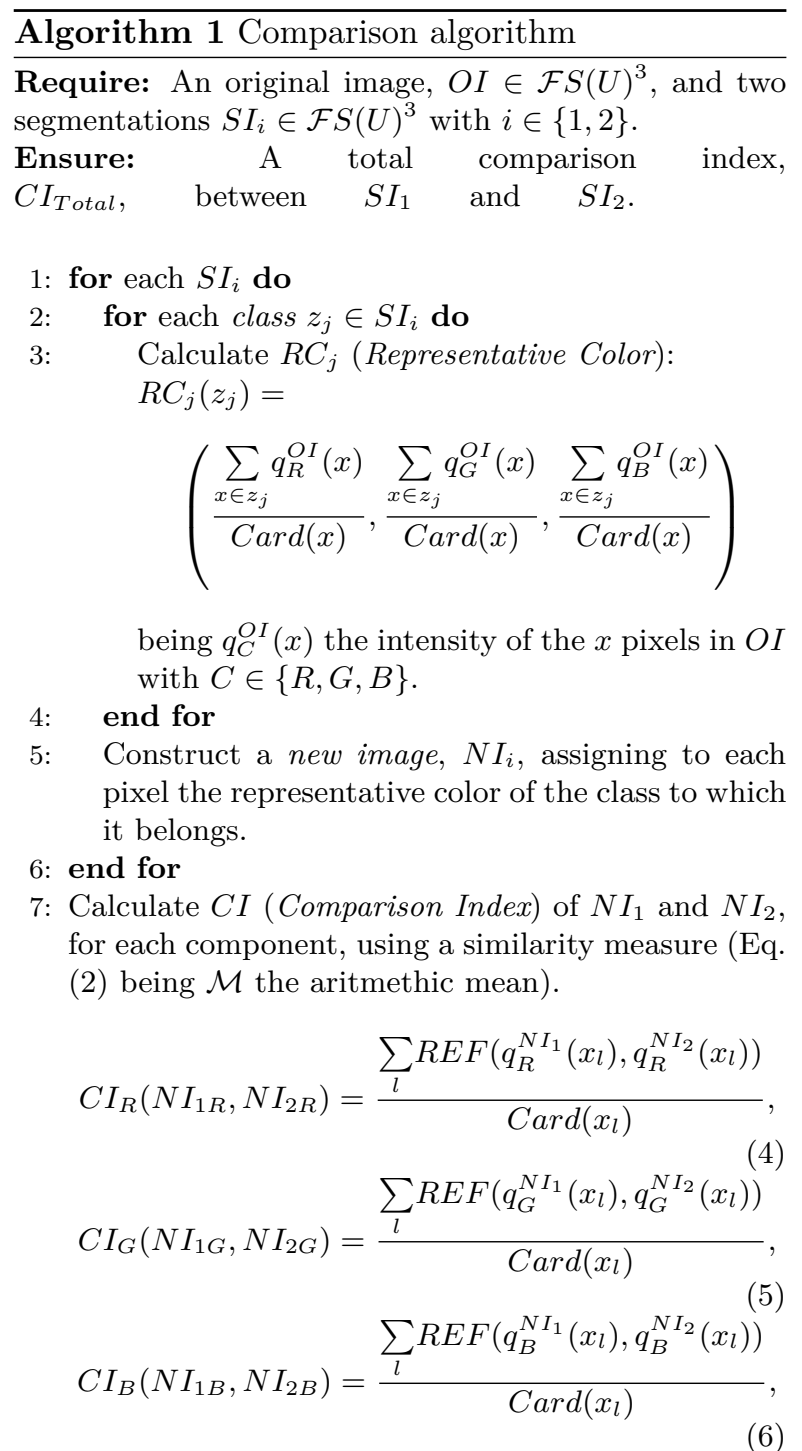

being $q_{C}^{N I_{i}}\left(x_{l}\right)$ the intensity of the $x_{l}$ pixels of $N I_{i} \in$ $\left\{N I_{1}, N I_{2}\right\}$ with $C \in\{R, G, B\}$.

8: Calculate IC Total (Total Comparison Index) aggregating the comparison index of each component

$$
\begin{gathered}
C I_{\text {Total }}=\frac{1}{3} \cdot\left(C I_{R}\left(N I_{1 R}, N I_{2 R}\right)+\right. \\
\left.C I_{G}\left(N I_{1 G}, N I_{2 G}\right)+C I_{B}\left(N I_{1 B}, N I_{2 B}\right)\right) .
\end{gathered}
$$

\section{Experimental results}

In the experiment we have used 20 images from the Berkeley Segmentation Dataset (BSDS) [17], containing 100 images. All the images in the subset have a resolution of $321 \times 481(434 \times 291)$ pixels and 
are provided in RGB color space. This database also provides different ideal segmentations for every image.

To obtain the segmented images, we use three different segmentations approaches: the classical Fuzzy C-Means (FCM) [2], the well-known maximum a posteriori (MAP) estimation and the maximum likelihood (ML) estimation [11] and the model based on 2-tuples (2-TUP) [21].

We also set up the number of zones into which the three algorithms segment the pixels. In 2TUP the pixels are always classified in six zones. Therefore, we parameterize FCM for six zones, too, and MAP-ML starting from six zones, instead of ten, as it is done by default. The ideal images have been selected with a number of zones greater than two and less than six (if possible). In this way, we ensure that the studied segmentation methods are not disadvantaged compared to segmentation carried out by humans.

In Fig. 1 we show the segmentations performed considering the previous approaches, for 10 of the images of the experiment. In the first column we show the original images with their identifiers in BSDS, in the second column the ideal images appear and in the third, fourth and fifth columns we show the segmentations obtained with 2-TUP, FCM and MAP-ML, respectively.

In this experiment we intend to quantify the performance of our approach (Algorithm 1), using Eq.(1) to calculate Eqs.(4)-(6), for comparing segmented color images. In order to do so, we compare our results with those obtained by one of the most used metrics, the Probabilistic Rand Index.

\subsection{Probabilistic rand index}

Rand Index [23] (see also [17]) is commonly used for the evaluation of segmented images when comparing with an ideal (hand-made segmentation) one. The main idea of this measure consists in counting the number of pairs of pixels that have a consistent labeling in both segmentations. It means, the number of pairs of pixels that in the ideal image and the evaluated images are labeled as the same object plus the number of pairs of pixels that in the ideal and evaluated images are labeled as different objects, divided by the total number of pairs of pixels of the image. The $R I$ is given by the following expression:

$$
\begin{aligned}
& R I(E v, I d)=\frac{1}{\left(\begin{array}{c}
N \times M \\
2
\end{array}\right)} \\
& \sum_{\substack{i, j \\
i \neq j}}\left[I\left(l_{e v}(i)=l_{e v}(j) \wedge l_{i d}(i)=l_{i d}(j)\right)\right. \\
& \left.\quad+I\left(l_{e v}(i) \neq l_{e v}(j) \wedge l_{i d}(i) \neq l_{i d}(j)\right)\right],
\end{aligned}
$$

where $E v$ is the evaluated image, $I d$ is the ideal image, $I$ is the identity function, $M \times N$ is the number of pixels in the image, $l_{e v}(i)$ represents each labeled pixel in $E v$ and $l_{i d}(i)$ represents each labeled pixel in $I d$.

The $R I$ ranges from 0 , when the two segmentations have no similarities (i.e. when one is a flat image and the other has every pixel segmented as a different class) to 1 , when the segmentations are identical.

\subsection{Obtained results}

We compare the segmented images obtained with 2-TUP, FCM and MAP-ML with the hand-made segmentations done by humans (these images are denoted as ideal images) using our proposal, Algorithm 1 (see Table 1), and the probabilistic rand index (see Table 2). In Algorithm 1 we have take Eq. (3) to calculate the Comparison Index of each component. In both tables, we highlight in bold the best result for every image.

\begin{tabular}{cccc}
\hline Image & $2-$ TUP & FCM & MAP-ML \\
\hline$(124084)$ & 0.9251 & 0.9058 & $\mathbf{0 . 9 5 2 2}$ \\
$(260058)$ & $\mathbf{0 . 9 6 2 5}$ & 0.9189 & 0.9496 \\
$(295087)$ & 0.9389 & 0.9035 & $\mathbf{0 . 9 4 0 5}$ \\
$(299086)$ & $\mathbf{0 . 9 7 7 0}$ & 0.9493 & $\mathbf{0 . 9 7 7 0}$ \\
$(161062)$ & $\mathbf{0 . 9 7 1 9}$ & 0.9613 & 0.9627 \\
$(207056)$ & 0.9692 & 0.9526 & $\mathbf{0 . 9 7 2 4}$ \\
$(374067)$ & 0.9662 & 0.9514 & $\mathbf{0 . 9 7 8 2}$ \\
$(67079)$ & 0.9429 & 0.9015 & $\mathbf{0 . 9 5 9 4}$ \\
$(100075)$ & $\mathbf{0 . 9 5 7 5}$ & 0.8930 & 0.9374 \\
$(58060)$ & 0.9460 & 0.9043 & $\mathbf{0 . 9 6 0 6}$ \\
$(216066)$ & 0.9275 & 0.8931 & $\mathbf{0 . 9 3 5 9}$ \\
$(291000)$ & $\mathbf{0 . 9 3 9 1}$ & 0.8743 & 0.9374 \\
$(295087)$ & 0.9389 & 0.9035 & $\mathbf{0 . 9 4 0 5}$ \\
$(80099)$ & 0.9868 & 0.9749 & $\mathbf{0 . 9 9 3 0}$ \\
$(253036)$ & 0.9464 & 0.9377 & $\mathbf{0 . 9 6 0 1}$ \\
$(100080)$ & 0.9268 & $\mathbf{0 . 9 5 2 9}$ & 0.9266 \\
$(197017)$ & 0.8503 & 0.9285 & $\mathbf{0 . 9 5 9 4}$ \\
$(46076)$ & 0.9239 & 0.9344 & $\mathbf{0 . 9 4 4 3}$ \\
$(118035)$ & $\mathbf{0 . 9 3 8 6}$ & 0.9244 & 0.93774 \\
$(126007)$ & $\mathbf{0 . 9 4 3 4}$ & 0.9160 & 0.9310 \\
$(69015)$ & 0.9333 & 0.9046 & $\mathbf{0 . 9 4 3 6}$ \\
\hline
\end{tabular}

Table 1: Total Comparison index between ideal image and segmentations given by 2-TUP (second column), FCM (third column) and MAP-ML(fourth column).

As we can see, our proposal and the $R I$ obtain very different results when comparing the same image segmentations. As we have said, Algorithm 1 is only based on the color of the created areas, while $R I$ is based in whether every pair of pixels are located in the same or in different area in both segmentations.

For example, if we analyze the seventh image (374067), we see that our algorithm ensures that the best segmentation is the one performed by MAPML while the $R I$ ensures that is the one obtained by 2 -TUP. The ideal segmentation is divided in more than ten areas, separating different parts of the grass, groups of trees, each person, etc. The segmentation obtained by 2-TUP distinguish 

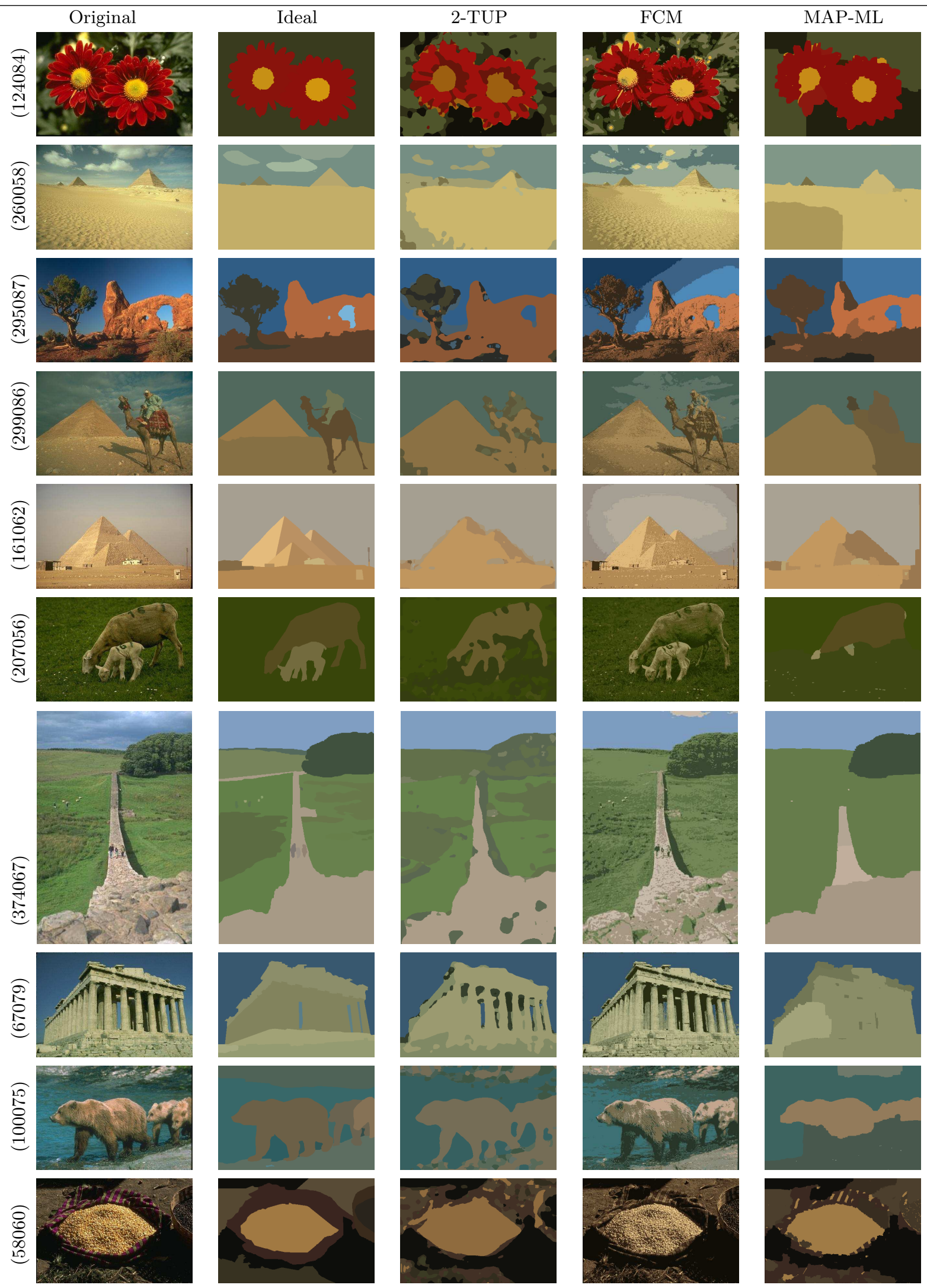

Figure 1: Original images (first column), ideal handmade segmentation (second column) and their segmentations with different approaches, 2-TUP (third column), FCM (fourth column) and MAP-ML (fifth column).

different areas in the grass, but it does not separate the area with big trees. On the other side, the segmentation by MAP-ML separates only big areas: all the grass is in the same area. But analyzing only 


\begin{tabular}{cccc}
\hline & $2-$ TUP & FCM & MAP-ML \\
\hline$(124084)$ & 0.7152 & 0.7134 & $\mathbf{0 . 7 6 0 0}$ \\
$(260058)$ & $\mathbf{0 . 8 0 6 3}$ & 0.6551 & 0.7461 \\
$(295087)$ & $\mathbf{0 . 8 2 6 9}$ & 0.7775 & 0.7995 \\
$(299086)$ & 0.8389 & 0.7718 & $\mathbf{0 . 8 6 6}$ \\
$(161062)$ & 0.8836 & 0.7197 & $\mathbf{0 . 9 1 6 2}$ \\
$(207056)$ & $\mathbf{0 . 6 4 5 3}$ & 0.5656 & 0.6343 \\
$(374067)$ & $\mathbf{0 . 8 6 3 3}$ & 0.8011 & 0.7559 \\
$(67079)$ & 0.7918 & $\mathbf{0 . 8 1 0 9}$ & 0.7646 \\
$(100075)$ & 0.7048 & 0.7446 & $\mathbf{0 . 7 7 2 7}$ \\
$(58060)$ & 0.8112 & 0.7319 & $\mathbf{0 . 8 3 3 3}$ \\
$(216066)$ & 0.7630 & 0.7127 & $\mathbf{0 . 7 7 8 7}$ \\
$(291000)$ & 0.7675 & 0.6981 & $\mathbf{0 . 7 7 1 3}$ \\
$(80099)$ & $\mathbf{0 . 6 1 9 2}$ & 0.5864 & 0.4855 \\
$(253036)$ & $\mathbf{0 . 7 7 5 4}$ & 0.7561 & 0.7481 \\
$(100080)$ & 0.7598 & $\mathbf{0 . 7 7 6 8}$ & 0.775 \\
$(197017)$ & 0.7115 & 0.7627 & $\mathbf{0 . 8 5 1 8}$ \\
$(46076)$ & 0.8430 & $\mathbf{0 . 8 5 7 8}$ & 0.8305 \\
$(118035)$ & 0.8807 & 0.8155 & $\mathbf{0 . 8 9 8 5}$ \\
$(126007)$ & 0.7873 & $\mathbf{0 . 8 6 2 1}$ & 0.8025 \\
$(69015)$ & $\mathbf{0 . 7 6 6 7}$ & 0.6997 & 0.6798 \\
\hline
\end{tabular}

Table 2: Probabilistic rand index between ideal image and segmentations given by 2 -TUP (second column), FCM (third column) and MAP-ML (fourth column).

the color, the grass has quite similar color, so the error of putting all them together is not so big. In the same sense, although the road is divided into three parts, all the representative colors are pretty similar, so the differences with the color of the road in the ideal segmentation are very low.

\section{Conclusions}

In this work we have presented an algorithm for the evaluation of segmented images. The methodology proposed consists in comparing the segmentation image obtained by any algorithm with the ideal (hand-made) segmentation. Contrary to evaluation methods in the literature that compare if the objects in both images are composed of the same pixels, our proposal measures the similarity between the average color of both objects. This work is the beginning of the definition of new comparison measures over lattices. In the near future it necessary to analyze the impact of applying these measures but considering other color spaces as well as other aggregate functions and restricted equivalence functions to calculate the comparison index.

\section{Acknowledgment}

This work was partially supported by the Research Services of the Universidad Publica de Navarra, and the National Science Foundation of Spain, references TIN2010-15055 and TIN2011-29520.

\section{References}

[1] P. Arbelaez, M. Maire, C. Fowlkes, J. Malik, Contour Detection and Hierarchical Image Segmentation, IEEE Trans. Pattern Anal. Mach. Intell., 33:898-916, 2011.

[2] J.C. Bezdek, R. Ehrlich and W. Full, FCM - The Fuzzy C-Means Clustering-Algorithm, Computers and Geosciences, 10:191-203, 1984.

[3] J.C. Bezdek, R. Chandrasekhar and Y. Attikiouzel ,A geometric approach to edge detection, IEEE Transactions on Fuzzy Systems, 6:52-75, 1998.

[4] J. C. Bezdek, J. Keller, R. Krisnapuram and N.R. Pal, Fuzzy Models and algorithms for pattern recognition and image processing, Kluwer, Boston, 1999.

[5] H. Bustince, E. Barrenechea and M. Pagola, Restricted equivalence functions, Fuzzy Sets and Systems, 157:2333-2346, 2006.

[6] H. Bustince, E. Barrenechea and M. Pagola, Image thresholding using restricted equivalence functions and maximizing the measures of similarity, Fuzzy Sets and Systems, 158:496-516, 2007.

[7] H. Bustince, E. Barrenechea, M. Pagola and J. Fernandez, Interval-valued fuzzy sets constructed from matrices: application to edge detection, Fuzzy Sets and Systems, 160:18191840, 2009.

[8] H. Bustince, M. Pagola, E. Barrenechea, J. Fernandez, P. Melo-Pinto, P. Couto, H. R. Tizhoosh, and J. Montero, Ignorance functions. An application to the calculation of the threshold in prostate ultrasound images, Fuzzy Sets and Systems, 161:20-36, 2010.

[9] T. Chaira, A.K. Ray, Region extraction using fuzzy similarity measures, Journal of Fuzzy Mathematics, 11:601-607, 2003.

[10] T. Chaira, A.K. Ray, Fuzzy measures for color image retrieval, Fuzzy Sets and Systems, 150:545-560, 2005.

[11] S. Chen, L. Cao, Y. Wang, J. Liu and $\mathrm{X}$. Tang, Image segmentation by MAP-ML estimations, IEEE Transactions on Image Processing, 19:2254-2264, 2010.

[12] J. Fan, W. Xie, Some Notes on Similarity Measure and Proximity Measure, Fuzzy Sets and Systems, 101:403-412, 1999.

[13] J. Fodor, M. Roubens, Fuzzy preference modelling and multicriteria decision support, Theory and Decision Library, Kluwer Academic, Dordrecht, 1994.

[14] F. Herrera and L. Martínez, A 2-tuple fuzzy linguistic representation model for computing with words, IEEE Transactions on Fuzzy Systems, 8:746-752, 2000.

[15] L. R. Liang and C. G. Looney, Competitive fuzzy edge detection, Applied Soft Computing, 3:123-137, 2003. 
[16] X. Liu, Entropy, Distance Measure and Similarity Measure of Fuzzy Sets and their Relations, Fuzzy Sets and Systems, 52:305-318, 1992.

[17] D. Martin, C. Fowlkes, D. Tal and J. Malik, A database of human segmented natural images and its application to evaluating segmentation algorithms and measuring ecological statistics, proceedings of the 8th International Conference on Computer Vision (Iccv 2001), pages 416-423, Vanvouver (Canada) 2001.

[18] M. Meila, Comparing clusterings: An axiomatic view, Proceedings of the 22nd international conference on Machine learning, pages 577-584, Bonn (Germany), 2005.

[19] E. Navon, O. Miller and A. Averbuch, Color image segmentation based on adaptive local thresholds, Image and Vision Computing, 23:69-85, 2005.

[20] M. Ozden, E. Polat, A color image segmentation approach for content-based image retrieval, Pattern Analysis, 43:3219-3232, 2007.

[21] R. Orduna, Overlap functions and their application to image segmentation, Ph. D. Thesis, Universidad Publica de Navarra, 2010.

[22] M.S. Prewitt, Object enhancement and extraction, A. Rosenfeld, B.S. Lipkin (Editors), Picture Processing and Psychophysics, Academic Press, NewYork, 1970.

[23] W. M. Rand, Objective criteria for the evaluation of clustering methods, Journal of the American Statistical Association, 66:846850, 1971.

[24] J. Sanz, A. Fernández, H. Bustince, and F. Herrera, A genetic tuning to improve the performance of fuzzy rule-based classification systems with interval-valued fuzzy sets: Degree of ignorance and lateral position, International Journal of Approximate Reasoning, 52:751-766, 2011.

[25] R. Unnikrishnan, C. Pantofaru, and M. Hebert, Toward objective evaluation of image segmentation algorithms, IEEE Transactions on Pattern Analysis and Machine Intelligence, 29: 929-944, 2007.

[26] D. Van der Weken, M. Nachtegael and E.E. Kerre, Using Similarity Measures and Homogeneity for the Comparison of Images, Image and Vision Computing, 22:695-702, 2004.

[27] X. Wang, B. De Baets and E. Kerre, A comparative Study of Similarity Measures, Fuzzy Sets and Systems, 73:259-268, 1995.

[28] L.A. Zadeh, Fuzzy sets, Information Control, 8:338-353, 1965. 